\title{
BMJ Open Effect of multimorbidity on survival of patients diagnosed with heart failure: a retrospective cohort study in Singapore
}

\author{
Palvinder Kaur, ${ }^{1}$ Nakul Saxena, ${ }_{1}^{1}$ Alex Xiaobin You, ${ }^{1}$ Raymond C C Wong, ${ }^{2}$ \\ Choon Pin Lim, ${ }^{3}$ Seet Yoong Loh, ${ }^{4}$ Pradeep Paul George ${ }^{1}$
}

To cite: Kaur P, Saxena N, You AX, et al. Effect of multimorbidity on survival of patients diagnosed with heart failure: a retrospective cohort study in Singapore. BMJ Open 2018;8:e021291. doi:10.1136/ bmjopen-2017-021291

- Prepublication history for this paper is available online. To view these files, please visit the journal online (http://dx.doi. org/10.1136/bmjopen-2017021291).

Received 21 December 2017 Revised 17 April 2018 Accepted 20 April 2018

Check for updates

${ }^{1}$ Health Services and Outcomes Research, National Healthcare Group, Singapore

2Department of Cardiology,

National University Heart Centre, Singapore, Singapore

${ }^{3}$ Department of Cardiology, National Heart Centre Singapore, Singapore

${ }^{4}$ Department of Cardiology, Tan Tock Seng Hospital, Singapore

Correspondence to Dr Pradeep Paul George: pradeep_paul_g_gunapal@nhg. com.sg

\section{ABSTRACT}

Objective Multimorbidity in patients with heart failure (HF) results in poor prognosis and is an increasing public health concern. We aim to examine the effect of multimorbidity focusing on type 2 diabetes mellitus (T2DM) and chronic kidney disease (CKD) on all-cause and cardiovascular disease (CVD)-specific mortality among patients diagnosed with $\mathrm{HF}$ in Singapore.

Design Retrospective cohort study.

Setting Primary and tertiary care in three (out of six) Regional Health Systems in Singapore.

Participants Patients diagnosed with HF between 2003 and 2016 from three restructured hospitals and nine primary care polyclinics were included in this retrospective cohort study.

Primary outcomes All-cause mortality and CVD-specific mortality.

Results A total of 34460 patients diagnosed with HF from 2003 to 2016 were included in this study and were followed up until 31 December 2016. The median followup time was 2.1 years. Comorbidities prior to HF diagnosis were considered. Patients were categorised as (1) HF only, (2) T2DM+HF, (3) CKD+HFand (4) T2DM+CKD+HF. Cox regression model was used to determine the effect of multimorbidity on (1) all-cause mortality and (2) CVDspecific mortality. Adjusting for demographics, other comorbidities, baseline treatment and duration of T2DM prior to HF diagnosis, 'T2DM+CKD+HF' patients had a $56 \%$ higher risk of all-cause mortality (HR: $1.56,95 \% \mathrm{Cl}$ 1.48 to 1.63 ) and a $44 \%$ higher risk of CVD-specific mortality (HR: $1.44,95 \% \mathrm{Cl} 1.32$ to 1.56) compared with patients diagnosed with $\mathrm{HF}$ only.

Conclusion All-cause and CVD-specific mortality risks increased with increasing multimorbidity. This study highlights the need for a new model of care that focuses on holistic patient management rather than disease management alone to improve survival among patients with HF with multimorbidity.

\section{INTRODUCTION}

With an ageing population and increased exposure to environmental risk factors, the likelihood of accumulating multiple chronic conditions has increased. ${ }^{12}$ Multimorbidity, defined as the coexistence of two or more chronic conditions, is an emerging epidemic in ageing populations around the world. ${ }^{3}$

\section{Strengths and limitations of this study}

This is the first study in Singapore that assessed a large proportion of patients with heart failure (HF) and the effect of multimorbidity on mortality among these patients.

- Due to the increasing prevalence of type 2 diabetes mellitus (T2DM) and chronic kidney disease (CKD) in the community and their strong link to HF, patients were categorised into four groups based on history of CKD (stages 1-5) and T2DM prior to HF diagnosis.

- Longitudinal follow-up and linked data with several databases provided accurate estimates of the survival probabilities for patients with HF.

- Due to the administrative nature of the database, only routinely collected variables were used and hence certain clinical parameters that were not captured could not be analysed.

- Data were limited to patients who sought care at any of the central healthcare institutions captured by the Regional Health System database.

Empirical data from 17 international studies reported the prevalence of multimorbidity of individuals aged $\geq 75$ years old to range from $13 \%$ to $83 \% .^{2}$ In Singapore, $51.5 \%$ of the elderly, aged 60 years and above, were found to have two or more chronic conditions. ${ }^{4}$

Heart failure (HF) is highly prevalent among older adults and carries a very high burden of comorbidities. ${ }^{5-8}$ The lifetime risk of developing $\mathrm{HF}$ is one in five at the age of 40 years 9 . In the USA, an estimated 6.5 million adults have HF, with 960000 incident cases annually. ${ }^{9}$ This HF pandemic is also evident in Asia. Reports published in East Asian countries, including Singapore, found that the prevalence of HF ranged from $1.3 \%$ to $6.7 \%$, with prevalence increasing with age ${ }^{78}$ A 2017 systematic review aimed to assess the magnitude and impact of multimorbidity on clinical outcomes in older adults with cardiovascular diseases (CVD) in mostly Western and European cohorts. ${ }^{6}$ In terms of the magnitude of multimorbidity, the prevalence of having 
an additional comorbidity occurred in $10 \%-77 \%$ of persons, while the proportion of persons having four or more additional comorbidities ranged from $5 \%$ to $60 \%$. ${ }^{6}$ Type 2 diabetes mellitus (T2DM), chronic kidney disease (CKD), anaemia, chronic obstructive pulmonary disease (COPD) and dementia/cognitive impairment were the most common comorbidities reported in these studies. While numerous studies have focused on the effects of individual comorbidities, few studies have examined the impact of multiple comorbidities on mortality in patients with $\mathrm{HF}^{5}{ }^{510-12}$ A positive association was seen in the cumulative effect of comorbidities and risk of dying. Multimorbidity in patients with $\mathrm{HF}$ is of public health concern as these patients have adverse health outcomes, greater healthcare utilisation, decreased quality of life, increased polypharmacy and increased risk of mortality. ${ }^{12} 1011$ 13-19

T2DM and CKD are established risk factors for CVD and are significantly associated with increased mortality. ${ }^{19-25}$ Among patients with chronic HF in Western populations, the prevalence of T2DM was approximately $25 \%$, while the prevalence of CKD was found to be at $50 \% .{ }^{26}{ }^{27}$ This triad of CVD, T2DM and CKD often coexist, has significant impact on mortality and are key comorbidities associated with an ageing population. ${ }^{19-21} 28$ These diseases share many similar risk factors, such as older age, obesity, physical inactivity, dyslipidaemia and hypertension. ${ }^{19-21} 29$ There is limited evidence on the effect of these comorbidities on the risk of mortality in patients with HF in Singapore. This study aims to determine the effect of multimorbidity, focusing on T2DM and CKD, on (1) all-cause and (2) CVD-specific mortality among patients diagnosed with HF in Singapore.

\section{METHODS}

\section{Study population}

Patients diagnosed with HF between 2003 and 2016 from three restructured hospitals and nine primary care polyclinics were included in this retrospective cohort study and followed up until death or censor date (31 December 2016). Patient demographics and clinical and death data were obtained from the Regional Health System (RHS) administrative database. A detailed report about the set-up of this database can be found in previous publications. ${ }^{31}$ Briefly, the RHS database is a conglomeration of four isolated databases from three RHS in the central region of Singapore. It contains linked National Healthcare Group polyclinic visit records from nine National Healthcare Group Polyclinics, specialist outpatient clinic visit records, hospital discharge records from three restructured hospitals, chronic disease management system records and mortality records obtained from the national registry. ${ }^{30} 31$

Comorbidities such as T2DM and CKD prior to HF diagnosis were considered. The effects of individual comorbidity and the combined effects of comorbidities were examined. Patients were categorised based on history of CKD (stages 1-5) and T2DM prior to HF diagnosis.
To obtain a reference group of patients with HF only, patients diagnosed with T2DM and/or CKD after HF was diagnosed were excluded. Patients were categorised as follows:

1. HF patients only (reference group).

2. History of T2DM+HF.

3. History of $\mathrm{CKD}+\mathrm{HF}$.

4. History of T2DM+history of CKD+HF.

\section{Outcomes}

The primary endpoint was all-cause mortality. The secondary endpoint was a CVD-specific mortality that included death from a cardiovascular cause, such as myocardial infarction, $\mathrm{HF}$ and peripheral vascular disease.

\section{Statistical analysis}

Descriptive analysis was used to report baseline characteristics of the four categories of patients. Data were reported as mean $\pm \mathrm{SD}$ for continuous variables and column percentages for categorical variables. Baseline characteristics were compared between the patient categories using the parametric one-way analysis of variance for continuous variables and $\chi^{2}$ test for categorical variables. Kaplan-Meier survival curves were used to estimate survival time between patient categories for all-cause and CVD-specific mortality. Cox regression was used to determine independent effects of comorbidity as well as the combined effects of comorbidities on risk of all-cause and CVD-specific mortality. Variables included in the model were age, gender, ethnicity, smoking status, history of atrial fibrillation, COPD, dyslipidaemia, hypertension and stroke, baseline medication such as ACE inhibitors (ACEi), angiotensin receptor blockers (ARB), beta-blockers and diuretics, and duration of T2DM prior to HF diagnosis. All statistical tests were two-sided and a p value of smaller than 0.05 was considered significant for inclusion into the final multivariate model.

Years of potential life lost (YPLL) were estimated for patients in the four strata. YPLL provides an estimate of the average time a person would have lived if he or she had not died prematurely. YPLL was calculated by averaging the difference between the life expectancy based on the 2015 Singapore life tables and age at death for each patient in the four categories. ${ }^{32}$

All analyses were conducted using STATA V.15.0 statistical software.

\section{Patient and public involvement}

There was no patient and public involvement as this was a database research study.

\section{RESULTS}

\section{Patient demographics}

Patients diagnosed with HF from 2003 to 2016 were included in the analysis $(\mathrm{n}=34460)$. The median follow-up was 2.1 years (IQR: $0.5-4.9$ years). The mean age of these patients was 70.2 years (SD 14.0 years) and $46.6 \%$ were female. Prior to HF diagnosis, $50.5 \%$ had T2DM 
Table 1 Baseline demographics by combinations of comorbidities in patients with HF

\begin{tabular}{|c|c|c|c|c|c|}
\hline Characteristics & HF $(n=12880)$ & $\begin{array}{l}\text { HF+T2DM } \\
(n=7430)\end{array}$ & $\begin{array}{l}H F+C K D \\
(n=4186)\end{array}$ & $\begin{array}{l}\text { HF+T2DM+CKD } \\
(\mathrm{n}=9964)\end{array}$ & $P$ values \\
\hline Age at presentation for HF (years) (mean, SD) & $67.9(15.8)$ & $68.3(12.1)$ & $77.3(12.8)$ & $71.5(11.8)$ & $<0.01$ \\
\hline $\begin{array}{l}\text { Life expectancy from age at presentation for HF } \\
\text { (years) (mean, SD) }\end{array}$ & $87.7(3.8)$ & $87.3(3.1)$ & $90.2(4.0)$ & $88.1(3.3)$ & $<0.01$ \\
\hline Years of potential life lost (mean, SD) & $13.9(10.5)$ & $14.2(8.9)$ & $9.3(7.6)$ & $13.5(8.4)$ & $<0.001$ \\
\hline Charlson Comorbidity Index (mean, SD) & $2.80(3.30)$ & $5.01(3.76)$ & $5.59(3.70)$ & $6.95(3.61)$ & $<0.001$ \\
\hline \multicolumn{6}{|l|}{ Gender (col \%) } \\
\hline Female & 43.7 & 45.5 & 48 & 50.5 & \\
\hline Male & 56.3 & 54.5 & 52 & 49.5 & $<0.001$ \\
\hline \multicolumn{6}{|l|}{ Ethnicity (col \%) } \\
\hline Chinese & 70.6 & 62.5 & 80 & 65.8 & \\
\hline Malay & 13.8 & 17.2 & 11.2 & 17.1 & \\
\hline Indian & 8.2 & 15.1 & 4.6 & 12.4 & \\
\hline Others & 7.4 & 5.2 & 4.2 & 4.8 & $<0.001$ \\
\hline \multicolumn{6}{|l|}{ Smoking status (col \%) } \\
\hline Non-smokers & 16.4 & 29.9 & 35.9 & 47.6 & \\
\hline Ex-smokers & 10.9 & 13.3 & 17.8 & 18.4 & \\
\hline Smoking & 17 & 15.4 & 13.1 & 12 & \\
\hline Unknown & 55.7 & 41.4 & 33.2 & 22 & $<0.001$ \\
\hline \multicolumn{6}{|l|}{ Previous comorbidity (col \%) } \\
\hline Atrial fibrillation & 6.6 & 9 & 19.2 & 13.3 & \\
\hline COPD & 4.1 & 4.3 & 8.2 & 4.6 & $<0.001$ \\
\hline Dyslipidaemia & 25.1 & 79 & 77.9 & 95.8 & $<0.001$ \\
\hline Hypertension & 26 & 77.3 & 77.5 & 94.6 & $<0.001$ \\
\hline Stroke & 6.6 & 14.3 & 20.6 & 22.3 & $<0.001$ \\
\hline \multicolumn{6}{|l|}{ Baseline medication (col \%) } \\
\hline ACEi & 7.5 & 27.1 & 23.4 & 33.8 & $<0.001$ \\
\hline ARB & 2.6 & 9.7 & 13.8 & 22.3 & $<0.001$ \\
\hline Beta-blockers & 13.6 & 29.8 & 41 & 46 & $<0.001$ \\
\hline Diuretics & 8.5 & 16.4 & 24.9 & 30.7 & $<0.001$ \\
\hline \multicolumn{6}{|l|}{ Mortality (col \%) } \\
\hline All-cause mortality & 50.1 & 57.7 & 60.2 & 58.4 & \\
\hline CVD-specific mortality & 21.5 & 26.4 & 23.2 & 24.3 & \\
\hline Non-CVD-specific mortality & 28.6 & 31.3 & 37 & 34.1 & $<0.001$ \\
\hline
\end{tabular}

ACEi, ACE inhibitors; ARB, angiotensin receptor blocker; CKD, chronic kidney disease; col \%, column percentages; COPD, chronic obstructive pulmonary disease; CVD, cardiovascular disease; HF, heart failure; T2DM, type 2 diabetes mellitus.

and $41.1 \%$ had CKD. The prevalences of other comorbidities were as follows: dyslipidaemia, $63.3 \%$; hypertension, $63.1 \%$; stroke, $14.5 \%$; atrial fibrillation, $10.6 \%$; and COPD, $4.8 \%$. A third of the patients in this cohort were non-smokers $(30.7 \%)$, while $14.7 \%$ were reported to be current smokers. Ex-smokers and patients whose smoking status was unknown made up the remaining proportion. Beta-blockers were the most commonly prescribed to this cohort (29.8\%), followed by ACEi (21.2\%), diuretics $(18.6 \%)$ and ARB (11.2\%).

Table 1 details the baseline characteristics of patients categorised according to their multimorbidity status.
A total of 12880 patients were diagnosed with HF only $(37.4 \%), 7430(21.6 \%)$ had T2DM+HF, $4186(12.1 \%)$ had CKD+HF and 9964 (28.9\%) patients had T2DM+CK$\mathrm{D}+\mathrm{HF}$. $\mathrm{CKD}+\mathrm{HF}$ patients were significantly older as compared with patients in the other three groups (table 1). T2DM+CKD+HF patients had higher prevalence of hypertension, dyslipidaemia and stroke as compared with other patients in this study (table 1). Nearly half of $\mathrm{T} 2 \mathrm{DM}+\mathrm{CKD}+\mathrm{HF}$ patients were on beta-blockers, while $34 \%$ were on ACEi.

The average YPLL for a T2DM+HF patient was 14.2 years (SD 8.9 years), while the average YPLL for a 
Table 2 Independent effect of diabetes and CKD on allcause and CVD-specific mortality

\begin{tabular}{|c|c|c|c|c|}
\hline & \multicolumn{2}{|c|}{$\begin{array}{l}\text { Primary endpoint* } \\
\text { All-cause mortality }\end{array}$} & \multicolumn{2}{|c|}{$\begin{array}{l}\text { Secondary endpointt } \\
\text { CVD-specific mortality }\end{array}$} \\
\hline & HR & $95 \% \mathrm{Cl}$ & HR & $95 \% \mathrm{Cl}$ \\
\hline T2DM & 1.21 & 1.17 to 1.26 & 1.21 & 1.14 to 1.28 \\
\hline CKD & 1.29 & 1.25 to 1.34 & 1.19 & 1.13 to 1.26 \\
\hline
\end{tabular}

*Adjusted for age, gender, ethnicity, smoking status, history of atrial fibrillation, COPD, dyslipidaemia, hypertension and stroke, baseline medication (ARB, beta-blockers and diuretics), and duration of T2DM prior to HF diagnosis.

†Adjusted for age, gender, ethnicity, smoking status, history of atrial fibrillation, COPD, dyslipidaemia, hypertension and stroke, baseline medication (ARB, ACEi and diuretics), and duration of T2DM prior to HF diagnosis.

ACEi, ACE inhibitor; ARB, angiotensin receptor blocker; CKD, chronic kidney disease; COPD, chronic obstructive pulmonary disease; CVD, cardiovascular disease; HF, heart failure; T2DM, type 2 diabetes mellitus.

CKD+HF patient was 9.3 years (SD 7.6 years) (table 1). As patients with $\mathrm{CKD}+\mathrm{HF}$ were significantly older than the other patients in this study, their YPLL was expected to be shorter.

\section{Mortality outcomes}

During the study period, there were 19083 (55.4\%) deaths, of which 8094 (23.5\%) were CVD-specific. As compared with other patient groups in the study, a higher proportion of CKD+HF patients $(60.2 \%)$ had died from an all-cause event (table 1). Interestingly, the proportion of patients experiencing a non-CVD-specific death was much higher than CVD-specific death across all four patient strata (table 1). CKD + HF patients had the highest proportion of non-CVD deaths $(37 \%)$, while only $28.6 \%$ of HF patients had a non-CVD-specific death (table 1).

Adjusting for demographics, other comorbidities, baseline treatment and duration of T2DM prior to HF diagnosis, the independent effects of T2DM and CKD showed a $21 \%$ (HR: $1.21,95 \%$ CI 1.17 to 1.26 ) and $29 \%$ (HR: $1.29,95 \%$ CI 1.25 to 1.34 ) higher risk of all-cause death as compared with patients with HF without these conditions (table 2). Likewise, the risk of CVD-specific death for T2DM and CKD was 21\% (HR: 1.21, 95\% CI 1.14 to 1.28 ) and $19 \%$ (HR: $1.19,95 \%$ CI 1.13 to 1.26 ) higher than patients with HF without these conditions (table 2).

Comorbidity was associated with a significantly increased risk of death among patients with increased level of comorbidities for both outcomes-all-cause mortality (figure 1) and CVD-specific death (figure 2). Adjusting for the same variables, the median survival to all-cause mortality for $\mathrm{HF}$ only, T2DM+HF, CKD+HF and T2DM+CKD+HF patients was 5.5, 4.6, 4.4 and 2.9 years, respectively. Having all three conditions (T2DM+CK$\mathrm{D}+\mathrm{HF}$ ) was associated with a 56\% (adjusted HR: 1.56, 95\% CI 1.48 to 1.63 ) higher risk of all-cause death and $44 \%$ (adjusted HR: 1.44, 95\% CI 1.32 to 1.56 ) higher risk

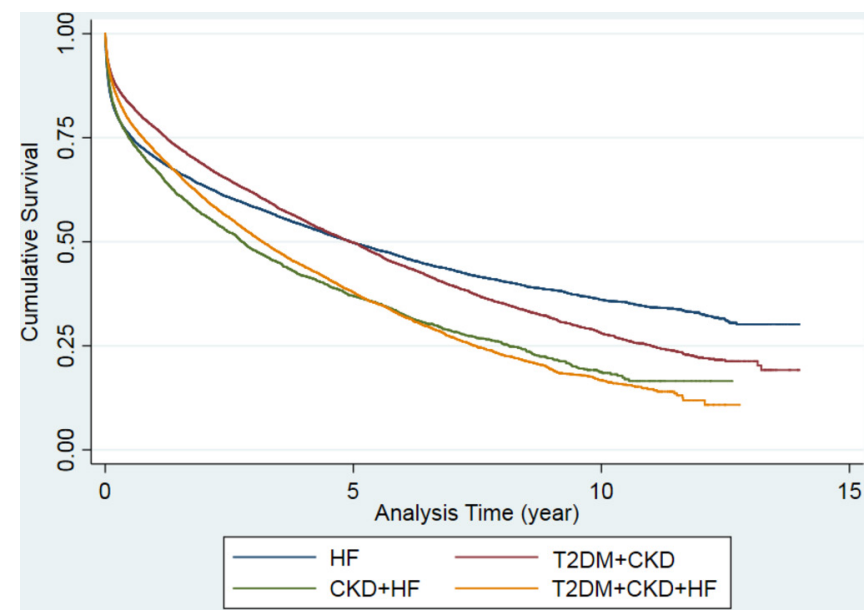

Figure 1 Kaplan-Meier survival plots for 'all-cause mortality' according to level of multimorbidity in patients with HF. CKD, chronic kidney disease; HF, heart failure; T2DM, type 2 diabetes mellitus.

from CVD-specific death compared with HF patients only (table 3) after adjustment of the specified confounders.

\section{DISCUSSION}

This study evaluated the effect of multimorbidity on survival of patients diagnosed with HF in Singapore. More than $40 \%$ of patients either had CKD, T2DM or both prior to developing HF. Findings from this study showed that presence of comorbidities among patients with HF increased the risk of all-cause mortality by $15 \%-56 \%$ and CVD-specific mortality by $17 \%-44 \%$.

The results from this study are in agreement with the findings from other published studies that assessed the effects of multimorbidity in patients diagnosed with CVD on risk of mortality. The European Heart Failure Pilot Survey consisting of 3226 outpatients with chronic HF reported that patients with three or more comorbidities had 9.3 times higher risk of death compared with patients

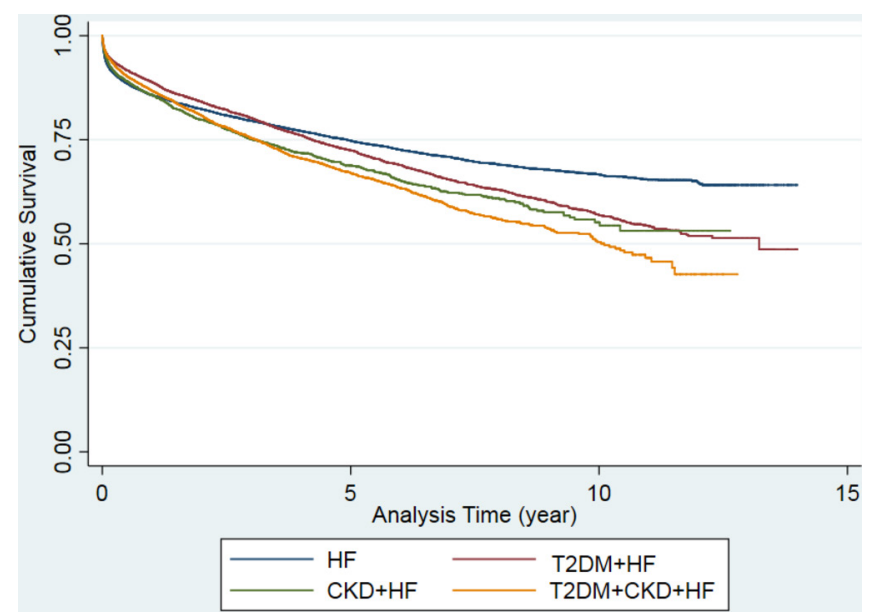

Figure 2 Kaplan-Meier survival plots for 'CVD-specific mortality' according to level of multimorbidity in patients with HF. CKD, chronic kidney disease; CVD, cardiovascular disease; HF, heart failure; T2DM, type 2 diabetes mellitus. 
Table 3 Effect of multimorbidity on all-cause and CVDspecific mortality

\begin{tabular}{|c|c|c|c|c|}
\hline & \multicolumn{2}{|c|}{$\begin{array}{l}\text { Primary endpoint* } \\
\text { All-cause mortality }\end{array}$} & \multicolumn{2}{|c|}{$\begin{array}{l}\text { Secondary endpoint } \\
\text { CVD-specific } \\
\text { mortality }\end{array}$} \\
\hline & HR & $95 \% \mathrm{Cl}$ & HR & $95 \% \mathrm{Cl}$ \\
\hline $\begin{array}{l}\text { HF (reference } \\
\text { group) }\end{array}$ & 1 & & 1 & \\
\hline $\mathrm{HF}+\mathrm{T} 2 \mathrm{DM}$ & 1.15 & 1.10 to 1.20 & 1.17 & 1.09 to 1.26 \\
\hline $\mathrm{HF}+\mathrm{CKD}$ & 1.20 & 1.14 to 1.27 & 1.14 & 1.05 to 1.24 \\
\hline $\mathrm{HF}+\mathrm{T} 2 \mathrm{DM}+\mathrm{CKD}$ & 1.56 & 1.48 to 1.63 & 1.44 & 1.32 to 1.56 \\
\hline
\end{tabular}

*Adjusted for age, gender, ethnicity, smoking status, history of atrial fibrillation, COPD, dyslipidaemia, hypertension and stroke, baseline medication (ARB, beta-blockers and diuretics), and duration of T2DM prior to HF diagnosis.

†Adjusted for age, gender, ethnicity, smoking status, history of atrial fibrillation, COPD, dyslipidaemia, hypertension and stroke, baseline medication (ARB, ACEi and diuretics), and duration of T2DM prior to HF diagnosis.

ARB, angiotensin receptor blocker; CKD, chronic kidney disease; COPD, chronic obstructive pulmonary disease; CVD, cardiovascular disease; HF, heart failure; T2DM, type 2 diabetes mellitus.

with $\mathrm{HF}$ with no comorbidities. ${ }^{10}$ In the same study, individual effects of CKD (adjusted HR: 1.5, 95\% CI 1.1 to 2.1), anaemia (adjusted HR: $1.7,95 \%$ CI 1.2 to 2.4 ) and T2DM (adjusted HR: 1.7, 95\% CI 1.3 to 2.4) conferred the highest risk on all-cause mortality. A cohort study of patients with CVD in the UK reported that patients diagnosed with T2DM and CKD had 4.7 times higher all-cause mortality risk and 2.7 times higher CVD-specific mortality risk as compared with patients diagnosed with HF only. ${ }^{33}$ This current study also showed that the presence of one or more comorbidities in patients with HF had a pronounced effect on all-cause mortality as compared with CVD-specific mortality.

Similar pathophysiology or risk factors shared by various comorbidities may increase the risk of mortality either by interacting with or aggravating the progression of HF. ${ }^{19-21} 29$ The interaction between T2DM, CKD and HF is a complex process-(1) they share common risk factors such as hypertension, dyslipidaemia, serum cholesterol and obesity; (2) the bidirectional pathophysiological and clinical effects of one disease can impact the other; and (3) pharmacological therapy for one disease may result in the development of the other, thus causing a clinical conundrum. ${ }^{19-21}$ 34-36 Poor glycaemic control is associated with increased risk of HF. With every $1 \%$ increase in glycosylated haemoglobin levels, there is an $8 \%$ increased risk of HF. ${ }^{37}$ T2DM increases risk of $\mathrm{HF}$ through a combination of pathophysiological abnormalities. ${ }^{38}$ T2DM alters the myocardial glucose metabolism, cardiac fatty acid metabolism and intracellular cycling. ${ }^{38}$ It also causes coronary artery disease, microvascular dysfunction and neurohormonal upregulation, and increases cardiac fibrosis. ${ }^{38} \mathrm{Clin}-$ ical studies have also shown that HF is an insulin-resistant state and could be responsible for the increased incidence of diabetes mellitus in this group of patients. ${ }^{38-41}$ Factors such as HF disease severity, decreased functional exercise capacity and increased B-type natriuretic peptide were found to be associated with greater insulin resistance. ${ }^{40} 41$ Furthermore, pharmacological treatments for HF such as diuretics and beta-blockers have been shown to be associated with an increase in incident T2DM..$^{38} 4041$

Renal dysfunction and HF share similar underlying comorbidities such as hypertension, T2DM and coronary arterial disease, and similar pathogenic mechanisms such as neurohormonal and inflammatory activation. ${ }^{20}{ }^{21}$ The bidirectional relationship of the heart-kidney interaction is termed as the cardiorenal syndrome, whereby the acute chronic dysfunction of one organ may induce an acute chronic dysfunction in the other. ${ }^{34} 3842$ While HF has shown to be a predictor of renal dysfunction, patients with CKD were reported to have up to $68 \%$ higher risk of developing $\mathrm{HF}$ as compared with patients without CKD. ${ }^{34}$ Decreased cardiac output, increased central venous pressure, several neurohormonal pathways such as the the renin-angiotensin-aldosterone system (RAAS) and HF therapies contribute to renal dysfunction in patients with $\mathrm{HF}^{34}{ }^{38} \mathrm{HF}$ therapies such as ACEi and ARB can affect renal function by decreasing the glomerular filtration rate through intrarenal mechanisms. ${ }^{35}$ Further research on the pathophysiological pathways for this triad of diseases may provide better understanding on how these comorbidities interact, as well as provide potential treatment targets to treat these diseases without impacting on the other.

Currently, there is no gold standard in assessing and characterising the burden of multimorbidity. Several approaches exist to measure multimorbidity, such as counting comorbidities, using comorbidity indices such as the Charlson Comorbidity Index or by grouping specific comorbidities together. ${ }^{6}{ }^{43}$ The ease of using disease counts makes them the most common approach to define multimorbidity. However, they do not account for the clinical severity of the various comorbid conditions or the underlying pathophysiology. Comorbidity indices provide a multimorbidity score by assigning weights to a range of diseases based on clinical severity. Although clinical severity is considered, summary scores lack the ability to adequately characterise the complexity of multimorbidity in patients with HF. Furthermore, such scores are challenging to apply and integrate in the clinical management of patients with HF.

As older patients with $\mathrm{HF}$ are more likely to have multiple comorbidities that influence prognosis, this study aimed to assess the combined prognostic effects of the selected comorbidities. Additional comorbidities and geriatric conditions beyond the scope of this study may contribute to poor prognosis of multimorbid patients with HF in Singapore; however T2DM and CKD were focused on mainly due to their increasing prevalence in the community and their strong link to HF. The major strength of this study is the large sample size and that the patients are reflective of a 'real-world setting'. Such 
complex patients, who would generally be excluded from clinical trials, are increasingly being seen in healthcare systems around the world and are being treated by general practitioners, geriatricians, cardiologists and other healthcare providers. Furthermore, the RHS database allowed for longitudinal tracking of patients captured in the system over time, thus providing accurate estimates of the survival probabilities of patients with HF with multimorbidity.

This study is not without limitations. Due to the administrative nature of the RHS database, data availability is limited to routinely captured administrative data. Patients may have been diagnosed with a disease prior to clinic presentation at one of the RHS healthcare providers. Likewise, treatment data might be under-represented in our database due to limited data availability. Other potential risk factors that could have influenced the results such as social, functional and cognitive factors, left ventricular ejection fraction (LVEF) and previous coronary revascularisation procedures were not captured in this system and therefore not included in the Cox regression model as potential confounders. ${ }^{12} 1620$ The database is limited to patients who sought treatment at any of the RHS healthcare providers in the central region of Singapore. Data on patients who sought care outside of this catchment area will not be captured by the database.

Multimorbidity poses numerous healthcare management challenges for patients diagnosed with HF. The accumulation of chronic conditions contributes to poorer prognosis, unnecessary healthcare utilisation, complex medical treatments, duplicative tests, fragmented care and competing priorities, which may impact overall care and outcomes of patients. Current clinical guidelines address the management of a single disease. ${ }^{44}$ Findings from this study support the need for a new model of care that focuses on holistic patient management rather than disease management alone to improve survival among patients with HF with multimorbidity. A systematic framework to support active management of $\mathrm{HF}$ and other concurrent comorbidities is urgently required. Key strategies that could potentially improve health outcomes in the management of multimorbid patients with $\mathrm{HF}$ include (1) acknowledging multimorbidity in $\mathrm{HF}$ as a clinical syndrome with poor prognosis, (2) determining the nature and extent of multimorbidity through routine clinical assessment, (3) identifying clinical priorities and appropriate care plans using a multidisciplinary team approach that includes patients and caregivers, (4) enhancing standard HF management guidelines to foster a patient-centred, multidisciplinary, case management strategy, and (5) evaluating the success of HF management that includes acute hospitalisation indicators as well as health-related indicators from the patient's perspectives such as the patient's goals and other patient-reported outcomes. ${ }^{45}$

\section{CONCLUSION}

The combined effects of T2DM and CKD resulted in the highest risk of mortality in patients with HF. The prevalence of HF, diabetes and CKD will continue to rise with the ageing population in Singapore. Optimal management of HF along with its associated multimorbidity is essential to improve survival. Pharmacological and non-pharmacological therapies are therefore required to prevent the development of these illnesses and reduce the combined effects of these diseases on morbidity and mortality in the population.

Acknowledgements We are thankful to the staff of the Department of Health Services and Outcomes Research, National Healthcare Group, Singapore, for their contributions in providing the data and valuable inputs to this study.

Contributors NS and PPG designed the study. PK conducted the data analysis and wrote the manuscript, with inputs from NS, PPG, LCP, RCCW and LSY. AXY provided biostatistics expertise and helped with data extraction from the database. RCCW, LCP and LSY provided clinical inputs for the study. All authors read and amended the manuscript, and all authors vetted the final version of the manuscript.

Funding This research received no specific grant from any funding agency in the public, commercial or not-for-profit sectors.

Competing interests None declared.

Patient consent Not required.

Ethics approval This study has been approved by the National Healthcare Group Domain Specific Review Board (NHG DSRB).

Provenance and peer review Not commissioned; externally peer reviewed. Data sharing statement № additional data available.

Open Access This is an Open Access article distributed in accordance with the Creative Commons Attribution Non Commercial (CC BY-NC 4.0) license, which permits others to distribute, remix, adapt, build upon this work non-commercially, and license their derivative works on different terms, provided the original work is properly cited and the use is non-commercial. See: http://creativecommons.org/ licenses/by-nc/4.0/

(c) Article author(s) (or their employer(s) unless otherwise stated in the text of the article) 2018. All rights reserved. No commercial use is permitted unless otherwise expressly granted.

\section{REFERENCES}

1. Palladino R, Tayu Lee J, Ashworth M, et al. Associations between multimorbidity, healthcare utilisation and health status: evidence from 16 European countries. Age Ageing 2016;45:431-5.

2. Salive ME. Multimorbidity in older adults. Epidemiol Rev 2013;35:75-83.

3. Almirall J, Fortin M. The coexistence of terms to describe the presence of multiple concurrent diseases. J Comorb 2013;3:4-9.

4. Picco L, Achilla E, Abdin E, et al. Economic burden of multimorbidity among older adults: impact on healthcare and societal costs. BMC Health Serv Res 2016;16:1-12.

5. Saczynski JS, Go AS, Magid DJ, et al. Patterns of comorbidity in older adults with heart failure: the Cardiovascular Research Network PRESERVE study. J Am Geriatr Soc 2013;61:26-33.

6. Tisminetzky M, Goldberg R, Gurwitz JH. Magnitude and Impact of Multimorbidity on Clinical Outcomes in Older Adults with Cardiovascular Disease: A Literature Review. Clin Geriatr Med 2016;32:227-46.

7. Guo Y, Lip GY, Banerjee A. Heart failure in East Asia. Curr Cardiol Rev 2013;9:112-22.

8. Shimokawa H, Miura M, Nochioka K, et al. Heart failure as a general pandemic in Asia. Eur J Heart Fail 2015;17:884-92.

9. Benjamin EJ, Blaha MJ, Chiuve SE, et al. Heart Disease and Stroke Statistics-2017 Update: A Report From the American Heart Association. Circulation 2017;135:e146-e603.

10. van Deursen VM, Urso R, Laroche C, et al. Co-morbidities in patients with heart failure: an analysis of the European Heart Failure Pilot Survey. Eur J Heart Fail 2014;16:103-11. 
11. Ahluwalia SC, Gross CP, Chaudhry SI, et al. Impact of comorbidity on mortality among older persons with advanced heart failure. $J$ Gen Intern Med 2012;27:513-9.

12. Oudejans I, Mosterd A, Zuithoff NP, et al. Comorbidity drives mortality in newly diagnosed heart failure: a study among geriatric outpatients. J Card Fail 2012;18:47-52.

13. Braunstein JB, Anderson GF, Gerstenblith G, et al. Noncardiac comorbidity increases preventable hospitalizations and mortality among medicare beneficiaries with chronic heart failure. Journal of the American College of Cardiology. Elsevier Masson SAS 2003;42:1226-33.

14. Rusinaru D, Saaidi I, Godard S, et al. Impact of chronic obstructive pulmonary disease on long-term outcome of patients hospitalized for heart failure. Am J Cardiol 2008;101:353-8.

15. Dunlay SM, Redfield MM, Weston SA, et al. Hospitalizations After Heart Failure Diagnosis. J Am Coll Cardiol 2009;54:1695-702.

16. Murad K, Goff DC, Morgan TM, et al. Burden of Comorbidities and Functional and Cognitive Impairments in Elderly Patients at the Initia Diagnosis of Heart Failure and Their Impact on Total Mortality: The Cardiovascular Health Study. JACC Heart Fail 2015;3:542-50.

17. Chamberlain AM, Alonso A, Gersh BJ, et al. Multimorbidity and the risk of hospitalization and death in atrial fibrillation: A populationbased study. Am Heart J 2017;185:74-84.

18. Wong CY, Chaudhry SI, Desai MM, et al. Trends in comorbidity, disability, and polypharmacy in heart failure. Am J Med 2011:124:136-43.

19. Suckling R, Gallagher H. Chronic kidney disease, diabetes mellitus and cardiovascular disease: risks and commonalities. J Ren Care 2012;38(Suppl 1):4-11.

20. van Deursen VM, Damman K, van der Meer P, et al. Co-morbidities in heart failure. Heart Fail Rev 2014;19:163-72.

21. Metra M, Zacà V, Parati G, et al. Cardiovascular and noncardiovascular comorbidities in patients with chronic heart failure. J Cardiovasc Med 2011;12:76-84.

22. Hirakawa Y, Ninomiya T, Kiyohara Y, et al. Age-specific impact of diabetes mellitus on the risk of cardiovascular mortality: An overview from the evidence for Cardiovascular Prevention from Observational Cohorts in the Japan Research Group (EPOCH-JAPAN). J Epidemiol 2017;27:123-9.

23. Wright RS, Reeder GS, Herzog CA, et al. Acute myocardial infarction and renal dysfunction: a high-risk combination. Ann Intern Med 2002;137:563-9.

24. Glynn LG, Reddan D, Newell J, et al. Chronic kidney disease and mortality and morbidity among patients with established cardiovascular disease: a West of Ireland community-based cohort study. Nephrology Dialysis Transplantation 2007;22:2586-94.

25. Dauriz M, Targher G, Temporelli PL, et al. Prognostic Impact of Diabetes and Prediabetes on Survival Outcomes in Patients With Chronic Heart Failure: A Post-Hoc Analysis of the GISSI-HF (Gruppo Italiano per lo Studio della Sopravvivenza nella Insufficienza Cardiaca-Heart Failure) Trial. J Am Heart Assoc 2017;6:e005156-15.

26. Dei Cas A, Khan SS, Butler J, et al. Impact of diabetes on epidemiology, treatment, and outcomes of patients with heart failure. JACC Heart Fail 2015;3:136-45.

27. Damman K, Valente MA, Voors AA, et al. Renal impairment, worsening renal function, and outcome in patients with heart failure: an updated meta-analysis. Eur Heart $J$ 2014;35:455-69.

28. Lozano R, Naghavi M, Foreman $\mathrm{K}$, et al. Global and regional mortality from 235 causes of death for 20 age groups in 1990 and 2010: a systematic analysis for the Global Burden of Disease Study 2010. Lancet 2012;380:2095-128.

29. Global Burden of Metabolic Risk Factors for Chronic Diseases Collaboration. Cardiovascular disease, chronic kidney disease, and diabetes mortality burden of cardiometabolic risk factors from 1980 to 2010: a comparative risk assessment. Lancet Diabetes Endocrinol 2014;2:634-47.

30. Saxena N, You AX, Zhu Z, et al. Singapore's regional health systems-a data-driven perspective on frequent admitters and cross utilization of healthcare services in three systems. Int $J$ Health Plann Manage 2017;32:36-49.

31. Gunapal PPG, Kannapiran P, Teow KL, et al. Setting up a regional health system database for seamless population health management in Singapore. Proceedings of Singapore Healthcare 2016;25:27-34.

32. Department of Statistics. Complete life tables 2015-2016 for singapore Resident Population. 2017:1-26 http://www.singstat. gov.sg/docs/default-source/default-document-library/publications/ publications_and_papers/births_and_deaths/lifetable15-16.pdf (accessed on 26 Oct 2017).

33. Glynn LG, Buckley B, Reddan D, et al. Multimorbidity and risk among patients with established cardiovascular disease: a cohort study. $\mathrm{Br} \mathrm{J}$ Gen Pract 2008;58:488-94.

34. Shiba N, Shimokawa H. Chronic kidney disease and heart failure-Bidirectional close link and common therapeutic goal. J Cardiol 2011;57:8-17.

35. Konstam MA. Renal function and heart failure treatment: when is a loss really a gain? Circ Heart Fail 2011;4:677-9.

36. Patel PA, Liang L, Khazanie P, et al. Antihyperglycemic Medication Use Among Medicare Beneficiaries With Heart Failure, Diabetes Mellitus, and Chronic Kidney Disease. Circ Heart Fail 2016;9:e002638.

37. Iribarren C, Karter AJ, Go AS, et al. Glycemic control and heart failure among adult patients with diabetes. Circulation 2001;103:2668-73.

38. Campbell P, Krim S, Ventura H. The Bi-directional Impact of Two Chronic Illnesses: Heart Failure and Diabetes - A review of the Epidemiology and Outcomes. Card Fail Rev 2015;1:8-10.

39. Nichols GA, Moler EJ. Cardiovascular disease, heart failure, chronic kidney disease and depression independently increase the risk of incident diabetes. Diabetologia 2011;54:523-6.

40. Flores-Le-Roux JA, Chillarón JJ, Benaiges D, et al. New-Onset Diabetes in Heart Failure Patients: A Complex Relationship with Clinical Implications. Journal of Cardiology and Therapy 2014;1:65-73.

41. Tousoulis D, Oikonomou E, Siasos G, et al. 1st Cardiology Department, University of Athens Medical School, "Hippokration" Hospital, Athens, Greece. Diabetes Mellitus and Heart Failure. European Cardiology Review 2014;9:37.

42. Granata A, Clementi A, Virzì GM, et al. Cardiorenal syndrome type 4: From chronic kidney disease to cardiovascular impairment. Eur $J$ Intern Med 2016;30:1-6.

43. Sharabiani MT, Aylin P, Bottle A. Systematic review of comorbidity indices for administrative data. Med Care 2012;50:1109-18.

44. Rajadurai J, Tse HF, Wang CH, et al. Understanding the Epidemiology of Heart Failure to Improve Management Practices: An Asia-Pacific Perspective. J Card Fail 2017;23:327-39.

45. Stewart S, Riegel B, Boyd C, et al. Establishing a pragmatic framework to optimise health outcomes in heart failure and multimorbidity (ARISE-HF): A multidisciplinary position statement. Int $J$ Cardiol 2016;212:1-10. 